\title{
Stromal characteristics are adequate prognosticators for recurrence risk in ductal carcinoma in situ of the breast
}

\author{
Mieke Van Bockstal ${ }^{\text {a, b, * }}$, Kathleen Lambein ${ }^{\mathrm{c}, \mathrm{d}}$, Ann Smeets ${ }^{\mathrm{d}}$, Laurence Slembrouck ${ }^{\mathrm{e}}$, \\ Patrick Neven ${ }^{\mathrm{e}, \mathrm{f}}$, Ines Nevelsteen ${ }^{\mathrm{d}}$, Caroline Weltens ${ }^{\mathrm{g}}$, Erik Van Limbergen ${ }^{\mathrm{g}}$, \\ Marie-Rose Christiaens ${ }^{\mathrm{d}}$, Chantal Van Ongeval ${ }^{\mathrm{h}}$, Hans Wildiers ${ }^{\mathrm{i}}$, Louis Libbrecht ${ }^{\mathrm{j}}$, \\ Giuseppe Floris ${ }^{k, 1}$ \\ a Department of Pathology, Erasmus MC, University Medical Center Rotterdam, P.O. Box 2040, 3000 CA, Rotterdam, the Netherlands \\ ${ }^{\mathrm{b}}$ Laboratory of Experimental Cancer Research, Department of Radiation Oncology and Experimental Cancer Research, Ghent University, De Pintelaan 185, \\ 9000 Ghent, Belgium \\ ${ }^{c}$ Department of Pathology, AZ St Lucas Hospital Ghent, Groenebriel 1, 9000 Ghent, Belgium \\ ${ }^{d}$ Department of Surgical Oncology, University Hospitals Leuven, Herestraat 49, 3000 Leuven, Belgium \\ e Department of Oncology, KUL University of Leuven, Herestraat 49, 3000 Leuven, Belgium \\ ${ }^{\mathrm{f}}$ Department of Gynecology and Obstetrics, University Hospitals Leuven, Herestraat 49, 3000 Leuven, Belgium \\ ${ }^{g}$ Department of Radiotherapy Oncology, University Hospitals Leuven, Herestraat 49, 3000 Leuven, Belgium \\ h Department of Radiology, University Hospitals Leuven, Herestraat 49, 3000 Leuven, Belgium \\ ${ }^{i}$ Department of Medical Oncology, University Hospitals Leuven, Herestraat 49, 3000 Leuven, Belgium \\ ${ }^{j}$ Department of Pathology, University Clinics St Luc, Hippokrateslaan 10, 1200 Sint-Lambrechts-Woluwe, Belgium \\ ${ }^{\mathrm{k}}$ Department of Pathology, University Hospitals Leuven, Herestraat 49, 3000 Leuven, Belgium \\ ${ }^{1}$ Department of Imaging and Pathology, Laboratory of Translational Cell \& Tissue Research, KUL University of Leuven, Herestraat 49 , 3000 Leuven, Belgium
}

\section{A R T I C L E I N F O}

\section{Article history:}

Received 25 September 2018

Received in revised form

2 November 2018

Accepted 6 November 2018

Available online $\mathrm{xxx}$

\section{Keywords:}

Ductal carcinoma in situ

Breast

Myxoid stroma

Stromal inflammation

Recurrence risk

\begin{abstract}
A B S T R A C T
Background: Ductal carcinoma in situ (DCIS) of the breast constitutes a heterogeneous group of nonobligate precursors for invasive breast cancer. To date, adequate risk stratification is lacking, which is presumed to result in overtreatment. We previously identified myxoid stromal architecture as a potential prognosticator for loco-regional recurrence. In the present study, we investigated the prognostic potential of stromal characteristics.

Methods: Hematoxylin and eosin stained slides from 211 DCIS patients were reviewed. The following histological features were dichotomously assessed: nuclear grade, DCIS architecture, presence of necrosis, intraductal calcifications, stromal inflammation and myxoid stromal architecture. Loco-regional recurrences constituted the primary endpoint. Results: Cox regression analysis showed that high nuclear grade, myxoid stromal architecture and moderate to extensive stromal inflammation were significantly associated with decreased recurrence-free survival, independent of radiotherapy. Based on these features, a combined risk score (CRS) was calculated, ranging from zero to three. A high CRS of three was associated with significantly shorter recurrence-free survival. Nineteen patients had a CRS of three, of which three relapsed (15.7\%), whereas only one out of 113 patients with a CRS of zero relapsed (0.9\%). Conclusions: We were able to validate our previously reported findings regarding the prognostic potential of myxoid periductal stroma in an independent DCIS patient cohort. A CRS based on nuclear grade, myxoid stromal architecture and stromal inflammation might facilitate discrimination of low risk from high risk patients. Consequently, the CRS may tailor adjuvant therapy. Future research should investigate whether radiotherapy can be safely omitted in patients with a low CRS.
\end{abstract}

(c) 2018 Elsevier Ltd, BASO The Association for Cancer Surgery, and the European Society of Surgical Oncology. All rights reserved.

Abbreviations: BCS, breast-conserving surgery; CI, confidence interval; CRS, combined risk score; DCIS, ductal carcinoma in situ; HR, hazard ratio; ER, estrogen receptor; TILs, tumor-infiltrating lymphocytes.

* Corresponding author. Department of Pathology, Erasmus MC, P.O. Box 2040, 3000 CA, Rotterdam, the Netherlands.

E-mail address: m.vanbockstal@erasmusmc.nl (M. Van Bockstal). 


\section{Introduction}

Ductal carcinoma in situ (DCIS) constitutes a heterogeneous group of pre-invasive lesions, which are considered to be nonobligatory precursors of invasive breast cancer of no special type [1,2]. Despite decades of research, there are currently no unequivocal prognostic markers that allow adequate recurrence risk stratification and individualized treatment. Consequently, most patients who undergo breast-conserving surgery (BCS) are irradiated post-operatively [3]. Adjuvant hormonal therapy is considered for patients with estrogen receptor-positive DCIS. Since the majority of patients is treated after diagnosis, data on natural behavior of DCIS are scarce. Most information on natural progression is derived from limited series of patients with unresected DCIS, either because of initial misdiagnosis, or because patients declined or were unfit for surgery [4-6]. Since autopsy studies have shown that DCIS is often present at the moment of non-breast cancer-related death, concerns have been raised about patients currently being over-treated [2].

Trials were initiated to investigate whether active surveillance of non-high grade DCIS is not inferior to current standards of care [7-9]. Inclusion based on nuclear grade might be a possible weakness of these non-inferiority trials, as it was recently shown that low and intermediate grade DCIS diagnosed on core biopsy yield a significant chance of being upgraded towards invasive cancer after subsequent surgery [10]. Patients with unresected DCIS were shown to have substantially increased risks for developing ipsilateral invasive breast cancer, regardless the nuclear grade of the initial DCIS [4-6]. The median interval whereupon patients with low and intermediate grade DCIS develop invasive cancer, is generally longer than for patients with high grade DCIS [4]. Given the considerable life expectancy of most DCIS patients, we should not neglect this long-term relapse risk. We therefore hypothesize that active surveillance trials would benefit from additional prognostic markers to refine risk stratification. Nuclear grade might be considered a rough indicator of prognosis, but additional markers are required to identify patients who are truly at high risk for developing invasive breast cancer. Ideally, risk stratification should be based on multiple robust and reproducible histopathological features.

We previously identified myxoid stromal architecture as a promising prognostic marker for loco-regional recurrence [11]. In the present study, we validated the prognostic potential of myxoid stroma in a larger, independent DCIS patient cohort. We previously determined specific cut-offs for dichotomous assessment of histopathological features in DCIS, which resulted in high inter-observer concordance among thirteen pathologists [12]. We applied these cut-offs for 2-tier assessment in the present study to explore their value in recurrence risk stratification.

\section{Materials \& methods}

\section{Patients and tissue specimens}

Retrospective investigations were performed on a cohort of 462 consecutive patients diagnosed with pure DCIS. All women underwent breast-conserving surgery (BCS; i.e. lumpectomy) at the Leuven University Hospitals (Leuven, Belgium) between January 1st' 2000 and December 31st' 2009. Follow-up and patient data included the following: radiotherapy, hormonal therapy, DCIS size, margin status, estrogen receptor (ER) status, age at diagnosis, date of diagnosis, date of loco-regional recurrence. Margin status was classified into three categories according to the Van Nuys prognostic index [13]. Hormonal therapy encompassed treatment with selective ER-modulators or aromatase inhibitors of any duration.

Outcome included local (ipsilateral DCIS and/or invasive cancer) and regional recurrence (tumor in ipsilateral axillary, supraclavicular or internal mammary lymph nodes) [11,14,15]. Locoregional recurrence comprised lesions occurring at least 6 months after initial BCS $[11,14]$. Contralateral breast cancer was not considered as loco-regional recurrence. Information on outcome was retrieved from electronic patient reports. Inclusion in this study required minimum follow-up of 24 months, regardless the development of relapse within these first 24 months of follow-up (i.e. patients who recurred after the first six months but within the first 24 months of follow-up were not excluded). Exclusion criteria were follow-up $<24$ months, treatment by mastectomy or BCS followed by mastectomy within 6 months, male sex, a history of ipsilateral breast cancer and primary BCS elsewhere with surgery for subsequent recurrence in the Leuven University Hospitals (Figure A.1). All data were coded anonymously. This study was approved by the local ethics committee (S56265/ML10212) and complied with the REMARK Guidelines [16].

\section{Histopathological re-evaluation}

For each case, all hematoxylin and eosin stained slides were retrieved from the archives of the department of pathology, Leuven University Hospitals. All slides were reviewed and the following histopathological features were registered: nuclear grade, calcifications, necrosis, DCIS architecture, stromal inflammation and stromal architecture. Histopathological assessment was performed dichotomously, since we have previously shown that dichotomous assessment results in substantially lower interobserver variability [12]. Nuclear grade was assessed according to the ASCO/CAP protocol [17], and dichotomized as non-high versus high nuclear grade. Intraductal calcifications were scored as absent or present. Necrosis was categorized semi-quantitatively as absent or single cell necrosis versus any amount of comedonecrosis, since we have shown this cut-off is most robust for 2-tier assessment [12]. DCIS architecture was assessed as predominantly solid or non-solid, since predominantly $(\geq 50 \%)$ solid architecture was shown to be associated with increased recurrence risk [18]. Periductal stromal inflammation was assessed semiquantitatively as absent or mild versus moderate or extensive, as previously reported $[11,19]$. In the former, the stroma contains no or only few loosely arranged inflammatory cells without lymphoid aggregates (Figure A.2). In the latter, the periductal stroma contains aggregates of inflammatory cells, with or without lymphoid follicle formation. Periductal stromal architecture was classified dichotomously as $<33 \%$ or $\geq 33 \%$ of affected ducts surrounded by myxoid stroma, since we have previously shown that this cut-off is most robust [12]. Myxoid stroma was defined as previously described [20], i.e. loosely arranged collagen fibers interspersed with an amorphous, slightly basophilic substance. Sclerotic stroma has a regular dense appearance, similar to connective tissue distant of the lesion (Figure A.2) [11,20].

\section{Statistics}

Data were analyzed using IBM SPSS 25.0 software (Chicago, IL, USA). Descriptive statistics for all continuously assessed variables (patient age at diagnosis, DCIS size) were determined and included arithmetic mean, standard deviation, minimum and 
maximum value. Recurrence-free survival was defined as time from primary diagnosis to diagnosis of loco-regional recurrence (expressed in months) and constituted the primary endpoint. Follow-up was censored at the last clinical contact or at the date of (non-breast cancer-related) death. Kaplan-Meier survival plots were generated. Cox regression analysis was used to determine hazard ratios (HR) and 95\% confidence intervals (95\% CI) for all clinicopathological variables in relation to time to recurrence. All parameters were included in Cox multiple regression analysis with application of the stepwise backward method (i.e. consecutively excluding the variable with the highest p-value and broadest $95 \% \mathrm{CI}$, until only those variables remained that were significantly associated with recurrence-free survival). All clinicopathological features were included separately in models with correction for radiotherapy. Cumulative survival of these models was compared. All tests were two-sided and $\mathrm{p}<0.05$ was considered statistically significant.

\section{Results}

Patient and disease characteristics

According to the aforementioned inclusion criteria, 211 of 462 identified DCIS patients were eligible (Figure A.1). Median age at diagnosis was 56 (range 25-81). Median follow-up time amounted 124 months (range 24-199) for the entire cohort. Median disease-free survival was 125 months (range 24-199) for patients without recurrence and 54 months (range 12-161) for patients with recurrence. Nine patients $(4,3 \%)$ did not receive radiotherapy. Hormonal treatment was given to 128 patients $(62,7 \%)$. Information on irradiation and hormonal therapy was lacking for one patient. ER status was known in 146 patients (69,1\%), of which 122 (83,6\%) had ER-positive DCIS. Seven patients (3,3\%) developed ipsilateral local recurrence, of which two showed invasive $(0,9 \%)$ and five in situ recurrence $(2,4 \%)$. Ipsilateral regional lymph node metastases without local recurrence were not registered.

\section{Clinicopathological features and time to overall loco-regional recurrence}

Table 1 provides an overview of all clinicopathological features in relation to overall loco-regional recurrence. DCIS size and margin status were unknown in 6 and 11 patients, respectively. Age at diagnosis, DCIS size and margin status were not significantly associated with time to overall loco-regional recurrence $(p>0.05)$. Radiotherapy $(p=0.003)$ and hormonal treatment $(p=0.042)$

Table 1

Clinicopathological characteristics in relation to time to locoregional recurrence, as determined by single variable Cox regression survival analysis.

\begin{tabular}{|c|c|c|c|c|c|}
\hline & $\begin{array}{l}\text { No recurrence }(\mathrm{n}=204) \\
\text { Median (range) }\end{array}$ & $\begin{array}{l}\text { Recurrence }(\mathrm{n}=7) \\
\text { Median (range) }\end{array}$ & HR & $95 \% \mathrm{CI}$ & p-value \\
\hline \multirow[t]{2}{*}{ DCIS size $(\mathrm{mm})$} & $\begin{array}{l}57(25-81) \\
20(1-90)\end{array}$ & $\begin{array}{l}56(33-63) \\
36(20-40)\end{array}$ & $\begin{array}{l}0,953 \\
1,026\end{array}$ & $\begin{array}{l}0,880-1,032 \\
0,988-1,065\end{array}$ & $\begin{array}{l}0,238 \\
0,181\end{array}$ \\
\hline & No recurrence n (\%) & Recurrence n (\%) & HR & $95 \% \mathrm{CI}$ & p-value \\
\hline \multicolumn{6}{|l|}{ Radiotherapy } \\
\hline Irradiation & $196(97,5)$ & $5(2,5)$ & & & \\
\hline No irradiation & $7(77,8)$ & $2(22,2)$ & 12,697 & $2,312-69,740$ & $0,003^{*}$ \\
\hline Unknown & $1(100)$ & $0(0)$ & & & \\
\hline \multicolumn{6}{|l|}{ Hormonal therapy } \\
\hline Treated & $127(99,2)$ & $1(0,8)$ & & & \\
\hline Untreated & $76(92,7)$ & $6(7,3)$ & 9,026 & $1,082-75,319$ & $0,042^{*}$ \\
\hline Unknown & $1(100)$ & $0(0)$ & & & \\
\hline \multicolumn{6}{|l|}{ Estrogen receptor status } \\
\hline Positive & $121(99,2)$ & $1(0,8)$ & & & \\
\hline Negative & $22(91,7)$ & $2(8,3)$ & 11,576 & $1,047-128,032$ & $0,046^{*}$ \\
\hline Unknown & $61(93,8)$ & $4(6,2)$ & 5,255 & $0,565-48,861$ & 0,145 \\
\hline \multicolumn{6}{|l|}{ Margin status } \\
\hline$\geq 10 \mathrm{~mm}$ & $15(100)$ & $0(0)$ & & & \\
\hline$\overline{1}-9 \mathrm{~mm}$ & $108(98,2)$ & $2(1,8)$ & 0,000 & $<0,001-\infty$ & 0,991 \\
\hline$<1 \mathrm{~mm}$ & $72(96,0)$ & $3(4,0)$ & 0,502 & $0,083-3,040$ & 0,453 \\
\hline Unknown & $9(82,0)$ & $2(18,0)$ & & & \\
\hline \multicolumn{6}{|l|}{ Nuclear grade } \\
\hline Non-high & $140(98,6)$ & $2(1,4)$ & & & \\
\hline High & $64(92,8)$ & $5(7,2)$ & 5,392 & $1,043-27,886$ & $0,044^{*}$ \\
\hline \multicolumn{6}{|l|}{ Calcifications } \\
\hline Absent & $66(98,5)$ & $1(1,5)$ & & & \\
\hline Present & $138(95,8)$ & $6(4,2)$ & 2,671 & $0,321-22,204$ & 0,363 \\
\hline \multicolumn{6}{|l|}{ Necrosis } \\
\hline Absent/single cell & $93(98,9)$ & $1(1,1)$ & & & \\
\hline Focal/extensive & $111(94,9)$ & $6(5,1)$ & 5,365 & $0,644-44,676$ & 0,120 \\
\hline \multicolumn{6}{|l|}{ DCIS architecture } \\
\hline Non-solid & $129(96,3)$ & $5(3,7)$ & & & \\
\hline Solid & $75(97,4)$ & $2(2,6)$ & 0,747 & $0,143-3,892$ & 0,729 \\
\hline \multicolumn{6}{|l|}{ Stromal architecture } \\
\hline Sclerotic & $173(98,3)$ & $3(1,7)$ & & & \\
\hline Myxoid & $31(88,6)$ & $4(11,4)$ & 6,569 & $1,467-29,423$ & $0,014^{*}$ \\
\hline \multicolumn{6}{|l|}{ Stromal inflammation } \\
\hline Absent/mild & $149(98,7)$ & $2(1,3)$ & & & \\
\hline Moderate/extensive & $55(91,7)$ & $5(8,3)$ & 7,029 & $1,355-36,466$ & $0,020^{*}$ \\
\hline
\end{tabular}

CI: confidence interval; DCIS: ductal carcinoma in situ; HR: hazard ratio.

* Statistically significant. 


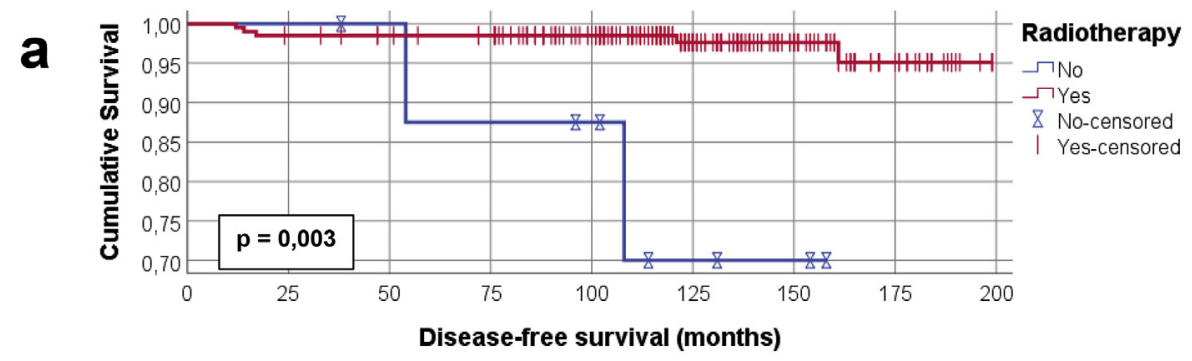

\begin{tabular}{|c|c|c|c|c|c|c|c|c|c|}
\hline No RT (n) & 9 & 9 & 8 & 7 & 6 & 3 & 2 & 0 & 0 \\
\hline RT $(n)$ & 201 & 197 & 193 & 189 & 162 & 97 & 52 & 26 & 0 \\
\hline
\end{tabular}

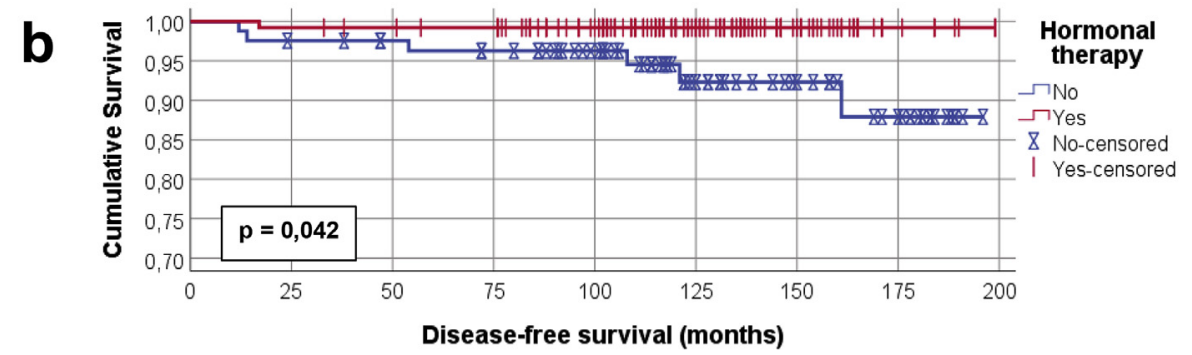

\begin{tabular}{|c|c|c|c|c|c|c|c|c|c|}
\hline No HT (n) & 82 & 79 & 76 & 73 & 63 & 36 & 25 & 17 & 0 \\
\hline HT (n) & 128 & 127 & 125 & 123 & 104 & 65 & 28 & 7 & 0 \\
\hline
\end{tabular}

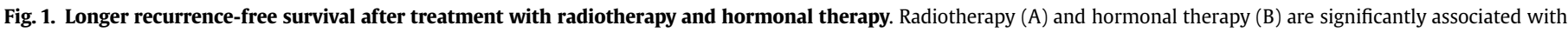

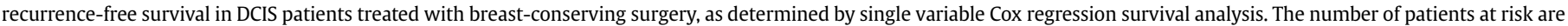
included below each Kaplan-Meier plot for each specified subgroup and for each time interval (in months).

were significantly associated with increased time to overall locoregional recurrence (Fig. 1). Intraductal calcifications, solid DCIS architecture and necrosis were not significantly related to time to overall loco-regional recurrence $(p>0.05)$. Moderate to extensive stromal inflammation $(\mathrm{p}=0.020)$, myxoid periductal stromal architecture $(p=0.014)$, high nuclear grade $(p=0.044)$ and ERnegativity $(p=0.046)$ were significantly associated with shorter time to loco-regional recurrence (Fig. 2). The limited number of overall recurrences precluded further analysis to investigate the prognostic potential of histopathological features for invasive or in situ recurrence only.

Cox multiple regression analysis and predictive modelling

Cox single variable regression analyses showed radiotherapy to be the strongest prognosticator for time to overall recurrence-free survival. Therefore, we aimed to investigate whether hormonal therapy, ER status, stromal inflammation, stromal architecture and nuclear grade remained significantly associated with time to overall recurrence after correction for irradiation (Table 2). After adjustment for radiotherapy, hormonal treatment was no longer associated with recurrence-free survival $(p>0.05)$. High nuclear grade $(p=0.043)$, ER-negativity $(p=0.020)$, moderate to extensive stromal inflammation $(\mathrm{p}=0.014)$ and myxoid stromal architecture $(\mathrm{p}=0.025)$ remained significantly associated with decreased recurrence-free survival, irrespective of radiotherapy.

Next, all clinicopathological characteristics significantly associated with time to loco-regional recurrence in Cox single regression analysis were entered into one model. Following the stepwise backward method, one variable at a time was excluded until a model was obtained in which each feature showed a statistically significant relationship with time to overall loco-regional recurrence. This model only comprised radiotherapy and stromal inflammation.

\section{Combined risk score}

Firstly, stromal architecture and stromal inflammation were combined into one 4-tier variable: 1 ) a reference category of absent to mild (i.e. low) stromal inflammation with sclerotic stroma, 2) low stromal inflammation with myxoid stroma, 3 ) moderate to extensive (i.e. high) stromal inflammation with sclerotic stroma, and 4) high stromal inflammation with myxoid stroma. Only the latter category was significantly associated with shorter recurrence-free survival when compared with the reference category, irrespective of radiotherapy (Table 3; Fig. 3A). ER status was not significantly associated with recurrence-free survival in this model.

Secondly, a combined risk score (CRS) was calculated, by adding the scores of nuclear grade, stromal architecture and stromal inflammation together. ER status was omitted from the CRS as it was unknown in $31 \%$ of this patient cohort. Non-high nuclear grade, absent to mild stromal inflammation and sclerotic stromal architecture were each scored as 0 . High nuclear grade, moderate to extensive stromal inflammation and myxoid stromal architecture were each scored as 1 . Scores were added up for each DCIS, resulting in a new 4 -tier variable named CRS, ranging from 0 to 3 . A CRS of zero was found in 113 patients, of which one relapsed $(0.9 \%)$. 
a

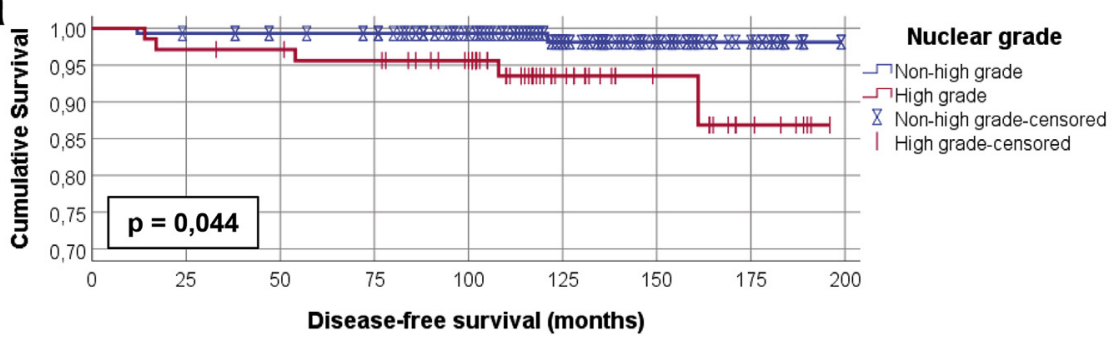

\begin{tabular}{|c|c|c|c|c|c|c|c|c|c|c|}
\hline $\mathrm{n}$ & 142 & 140 & 136 & 133 & 111 & 76 & 39 & 17 & 0 & Non-high grade \\
\hline $\mathrm{n}$ & 69 & 67 & 66 & 64 & 56 & 26 & 14 & 7 & 0 & High grade \\
\hline
\end{tabular}

b

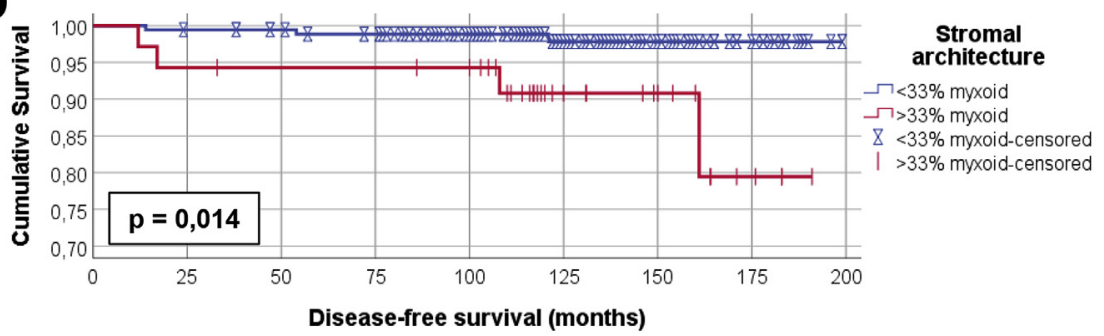

\begin{tabular}{|c|c|c|c|c|c|c|c|c|c|c|}
\hline $\mathrm{n}$ & 176 & 174 & 170 & 165 & 137 & 85 & 44 & 20 & 0 & $<33 \%$ myxoid \\
\hline $\mathrm{n}$ & 35 & 33 & 32 & 32 & 30 & 15 & 10 & 4 & 0 & $>33 \%$ myxoid \\
\hline
\end{tabular}

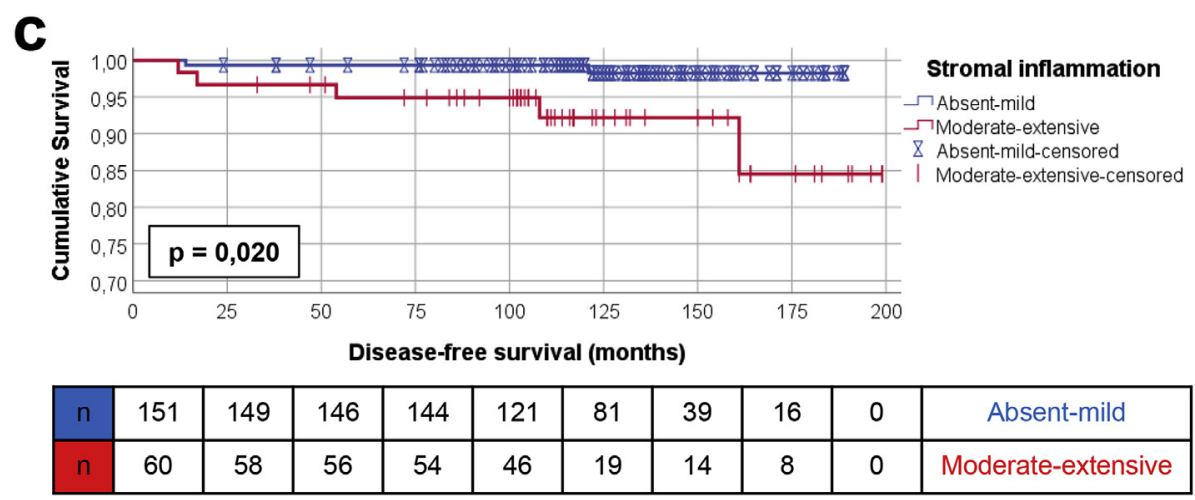

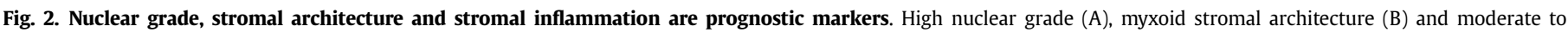

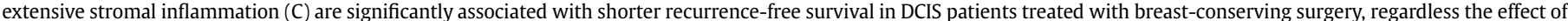

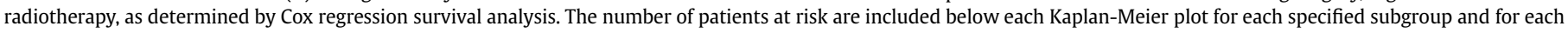
time interval (in months).

Fifty-one patients had a CRS of one, of which one relapsed $(2.0 \%$; 95\%CI 0,139-35,578). Twenty-eight patients had a CRS of two, of which two patients relapsed (7.1\%; 95\%CI 0,992-125,741). Nineteen patients had a CRS of three, of which three relapsed $(15.8 \%$; 95\%CI 1,699-163,304). Cox regression analysis without and with correction for radiotherapy was performed (Fig. 3B). A CRS of three was significantly associated with recurrence-free survival, regardless the effects of radiotherapy $(p=0.016)$. A CRS of one or two was not significantly associated with disease-free survival as compared with a CRS of zero, which was used as a reference category (Table 3).

\section{Discussion}

In this retrospective longitudinal cohort study of 211 BCStreated DCIS patients, we observed an overall low recurrence rate of $3.3 \%$ after a median follow-up of 124 months, which was likely due to radiotherapy since most patients were irradiated. Similar low long-term recurrence rates have been reported by others [21], and could be used as a guideline in future trials investigating noninferiority of alternative treatments.

Radiotherapy, nuclear grade, ER status, stromal architecture and stromal inflammation were identified as prognosticators for overall 
Table 2

Clinicopathological characteristics in relation to time to locoregional recurrence with adjustment for effects of radiotherapy, as determined by dual variable Cox regression survival analysis.

\begin{tabular}{|c|c|c|c|}
\hline & HR & $95 \% \mathrm{CI}$ & p-value \\
\hline Radiotherapy & 6,250 & $1,079-36,198$ & $0,041^{*}$ \\
\hline Hormonal therapy & 6,517 & $0,731-58,090$ & 0,093 \\
\hline Radiotherapy & 16,509 & $1,569-173,684$ & $0,020^{*}$ \\
\hline \multicolumn{4}{|l|}{ Margin status } \\
\hline$\overline{1}-9 \mathrm{~mm}$ & $<0,001$ & $<0,001-\infty$ & 0,991 \\
\hline$<1 \mathrm{~mm}$ & 0,387 & $0,059-2546$ & 0,323 \\
\hline Radiotherapy & 21,071 & $3,045-145,829$ & $0,002^{*}$ \\
\hline \multicolumn{4}{|l|}{ Estrogen receptor status } \\
\hline Negative & 20,661 & $1,604-266,192$ & $0,020^{*}$ \\
\hline Unknown & 5,036 & $0,548-46,319$ & 0,153 \\
\hline Radiotherapy & 13,281 & $2,360-74,737$ & $0,003^{*}$ \\
\hline Nuclear grade & 5,535 & $1,057-28,973$ & $0,043^{*}$ \\
\hline Radiotherapy & 13,773 & $2,474-76,689$ & $0,003^{*}$ \\
\hline Calcifications & 2,953 & $0,353-24,709$ & 0,318 \\
\hline Radiotherapy & 23,618 & $3,906-142,820$ & $0,001^{*}$ \\
\hline Necrosis & 8,622 & $0,959-77,532$ & 0,055 \\
\hline Radiotherapy & 12,819 & $2,327-70,620$ & $0,003^{*}$ \\
\hline DCIS architecture & 0,730 & $0,140-3,820$ & 0,710 \\
\hline Radiotherapy & 9,856 & $1,774-54,751$ & $0,009^{*}$ \\
\hline Stromal architecture & 5,627 & $1,239-25,552$ & $0,025^{*}$ \\
\hline Radiotherapy & 15,732 & $2,776-89,161$ & $0,002^{*}$ \\
\hline Stromal inflammation & 8,075 & $1,526-42,727$ & $0,014^{*}$ \\
\hline
\end{tabular}

CI: confidence interval; DCIS: ductal carcinoma in situ; HR: hazard ratio.

* Statistically significant. loco-regional recurrence. High nuclear grade, ER-negativity, myxoid stroma and moderate to extensive stromal inflammation were each significantly associated with shorter recurrence-free survival, irrespective of radiotherapy. We were therefore able to validate our previously reported findings in a larger, independent DCIS cohort [11]. Myxoid stroma is associated with lymph node metastasis and decreased disease-free survival in colorectal cancer [22,23], but it is a relatively unknown feature in breast cancer. Wernicke et al. reported a significant correlation between myxoid stroma and axillary lymph node metastasis in invasive breast cancer [24]. To our knowledge, no other studies on the prognostic role of myxoid stroma in DCIS have been reported, although myxoid periductal stroma in DCIS can often be perceived in microphotographs in a plethora of published reviews and studies [1,25-27]. It seems that myxoid stroma has been strikingly overlooked during the past decades, implying that conventional HE slides might yield more prognostic information than previously expected [20].

The role of stromal inflammation in breast cancer has been more intensively studied, although reports on tumor-infiltrating lymphocytes (TILs) in DCIS are limited. Pinder et al. reported a significant association between stromal inflammation and increased recurrence risk in DCIS, but detailed definitions for its assessment were omitted [18]. Contrariwise, Pruneri et al. were the first to provide a detailed definition of the assessment of TILs in an extensive DCIS cohort, but they were not able to demonstrate its prognostic value for recurrence risk [28]. However, this might have been due to the relatively shorter follow-up time or to differences in assessment, since TILs were quantified as a percentage according to the proposal of the International ImmunoOncology Biomarker Working Group [29]. Toss et al. compared seven different methods for assessment of TILs and demonstrated that evaluation as percentage was practically challenging and showed poor inter-observer concordance [30].

We previously observed substantial inter-observer concordance

Table 3

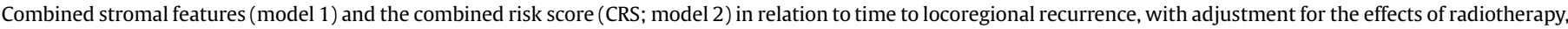
as determined by dual variable Cox regression survival analysis.

\begin{tabular}{|c|c|c|c|c|c|}
\hline & No recurrence $(\mathrm{n}=204) \mathrm{n}(\%)$ & Recurrence $(\mathrm{n}=7) \mathrm{n}(\%)$ & HR & $95 \% \mathrm{CI}$ & p-value \\
\hline \multicolumn{6}{|l|}{ MODEL 1} \\
\hline \multicolumn{6}{|l|}{ Radiotherapy } \\
\hline Irradiation & $196(97,5)$ & $5(2,5)$ & & & \\
\hline No irradiation & $7(77,8)$ & $2(22,2)$ & 14,329 & $2,499-82,156$ & $0,003^{*}$ \\
\hline Unknown & $1(100)$ & $0(0)$ & & & \\
\hline \multicolumn{6}{|l|}{ Combined stromal features } \\
\hline Sclerotic + low inflammation & $138(98,6)$ & $2(1,4)$ & & & \\
\hline Myxoid + low inflammation & $11(100)$ & $0(0)$ & 0,000 & $0,000-\infty$ & 0,988 \\
\hline Sclerotic + high inflammation & $35(97,2)$ & $1(2,8)$ & 2,683 & $0,238-30,219$ & 0,424 \\
\hline Myxoid + high inflammation & $20(83,3)$ & $4(16,7)$ & 11,967 & $2,165-66,133$ & $0,004^{*}$ \\
\hline \multicolumn{6}{|l|}{ MODEL 2} \\
\hline \multicolumn{6}{|l|}{ Radiotherapy } \\
\hline Irradiation & $196(97,5)$ & $5(2,5)$ & & & \\
\hline No irradiation & $7(77,8)$ & $2(22,2)$ & 13,004 & $2,276-163,304$ & $0,004^{*}$ \\
\hline Unknown & $1(100)$ & $0(0)$ & & & \\
\hline \multicolumn{6}{|l|}{ Combined risk score } \\
\hline Score 0 & $112(99,1)$ & $1(0,9)$ & & & \\
\hline Score 1 & $50(98,0)$ & $1(0,2)$ & 2,221 & $0,139-35,578$ & 0,573 \\
\hline Score 2 & $26(92,9)$ & $2(7,1)$ & 11,169 & $0,992-125,741$ & 0,051 \\
\hline Score 3 & $16(84,2)$ & $3(15,8)$ & 16,658 & $1,699-163,304$ & $0,016^{*}$ \\
\hline
\end{tabular}

CI: confidence interval; HR: hazard ratio.

* Statistically significant. 


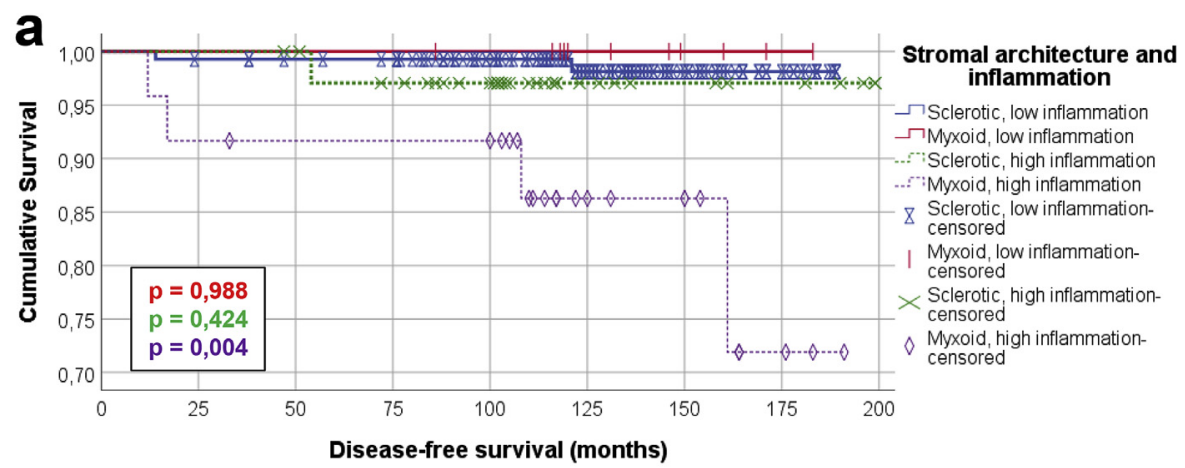

\begin{tabular}{|c|c|c|c|c|c|c|c|c|c|c|}
\cline { 2 - 12 } \multicolumn{1}{c|}{} & $\mathbf{0}$ & $\mathbf{2 5}$ & $\mathbf{5 0}$ & $\mathbf{7 5}$ & $\mathbf{1 0 0}$ & $\mathbf{1 2 5}$ & $\mathbf{1 5 0}$ & $\mathbf{1 7 5}$ & $\mathbf{2 0 0}$ & Months \\
\hline $\mathrm{n}$ & 140 & 138 & 135 & 133 & 111 & 76 & 36 & 15 & 0 & Sclerotic + low infl. \\
\hline $\mathrm{n}$ & 11 & 11 & 11 & 11 & 10 & 6 & 3 & 1 & 0 & Myxoid + low infl. \\
\hline $\mathrm{n}$ & 36 & 36 & 35 & 32 & 26 & 10 & 7 & 5 & 0 & Sclerotic + high infl. \\
\hline $\mathrm{n}$ & 24 & 22 & 21 & 21 & 20 & 9 & 7 & 3 & 0 & Myxoid + high infl. \\
\hline
\end{tabular}

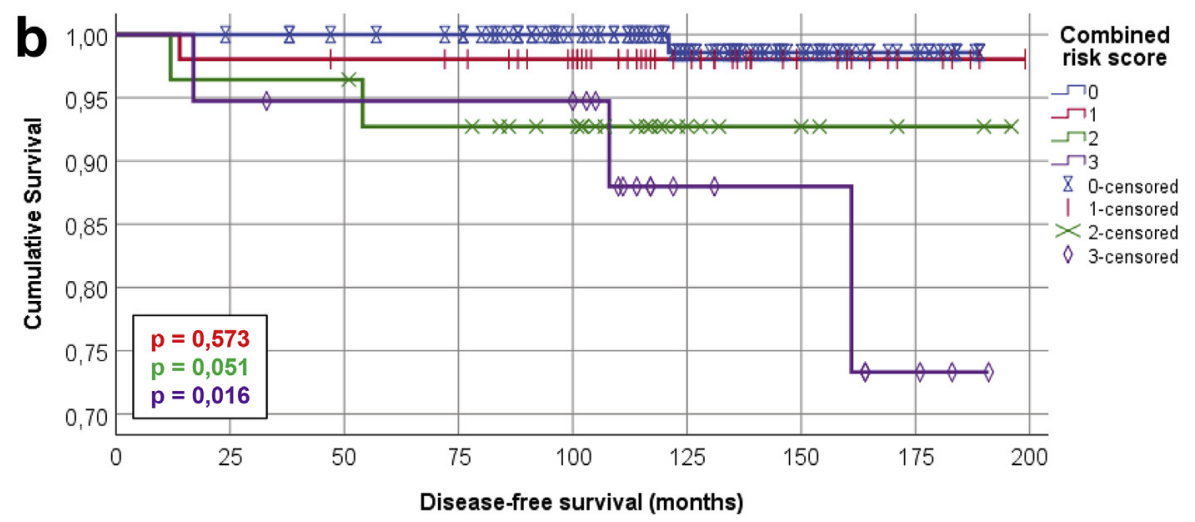

\begin{tabular}{|c|c|c|c|c|c|c|c|c|c|c|}
\cline { 2 - 12 } \multicolumn{1}{c|}{} & $\mathbf{0}$ & $\mathbf{2 5}$ & $\mathbf{5 0}$ & $\mathbf{7 5}$ & $\mathbf{1 0 0}$ & $\mathbf{1 2 5}$ & $\mathbf{1 5 0}$ & $\mathbf{1 7 5}$ & $\mathbf{2 0 0}$ & Months \\
\hline $\mathrm{n}$ & 113 & 112 & 109 & 107 & 88 & 61 & 31 & 13 & 0 & Score 0 \\
\hline $\mathrm{n}$ & 51 & 50 & 49 & 48 & 42 & 25 & 12 & 6 & 0 & Score 1 \\
\hline $\mathrm{n}$ & 28 & 27 & 27 & 25 & 21 & 7 & 4 & 2 & 0 & Score 2 \\
\hline $\mathrm{n}$ & 19 & 18 & 17 & 17 & 16 & 7 & 6 & 3 & 0 & Score 3 \\
\hline
\end{tabular}

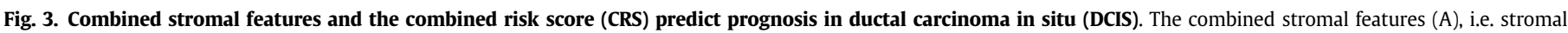

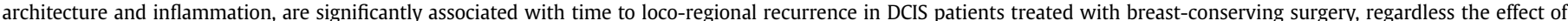

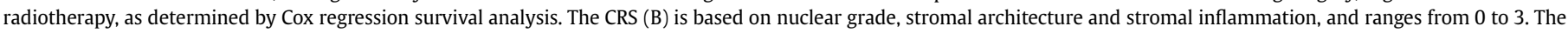

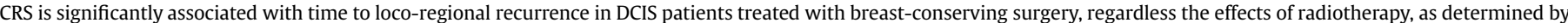

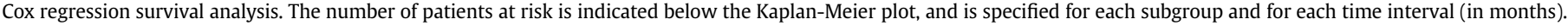

when stromal inflammation was assessed dichotomously [12]. From a scientific point of view, the major disadvantage of dichotomous histopathological assessment is the inherent loss of information. However, this tentative information loss does not outclass the advantage of high reproducibility of a risk stratification tool with clinical utility. After all, prognosticators for DCIS should be robust since they influence clinical decision-making. Ultimately, clinical decision-making is often dichotomous too (BCS versus mastectomy, post-operative irradiation versus no radiotherapy, etcetera), and would therefore benefit from a straightforward and robust aid for recurrence risk stratification.

Genomic and molecular studies provide an additional argument in favor of dichotomous histopathological assessment, since it is possible to discern a low- and high-grade pathway in breast cancer development [1,31,32]. Moreover, gene expression profiles corroborate 2-tier histopathological grading of DCIS, indicating a dichotomous grading scheme for DCIS at the molecular level [33]. There seems to be insufficient molecular evidence to support an "intermediate" grade among DCIS lesions. We therefore think the time is right to abandon 3-tier grading in DCIS and to adopt a 2-tier grading system, analogous to the binary grading schemes that are already used to classify gastro-intestinal dysplasia, non-invasive papillary urothelial carcinomas, and vulvar and cervical intraepithelial neoplasia (low-grade versus high-grade squamous intra-epithelial lesions).

Since we demonstrated here that 2-tier histopathological 
assessment can be used to discern low-risk from high-risk DCIS patients for overall recurrence, it would be worthwhile to further explore dichotomous risk stratification by validation of the CRS in larger cohorts, taking also into account hormone receptor status, DCIS size, resection margins and patient age. Although the latter are part of the Van Nuys prognostic index [13], we were not able to demonstrate their prognostic value in this cohort. This might have been due to the low number of overall recurrences in this cohort, or due to lack of power as DCIS size and margin status were not known for each patient.

The limited number of invasive recurrences in this cohort precluded further investigation of prognosticators for identification of patients at risk for developing invasive breast cancer. This remains one of the biggest challenges for future management of DCIS patients, especially when active surveillance might be considered [10]. It is of interest to investigate whether the CRS can discriminate patients at risk for invasive recurrence, since it has been shown that stromal inflammation in core biopsies with pure DCIS predicts the presence of invasive carcinoma in subsequent resection specimens [34-37]. Seventy-five percent of microinvasive carcinomas presents with stromal inflammation [38]. Moreover, Alexander et al. have shown that nuclear grade alone is probably insufficient to predict an associated invasive component when DCIS is diagnosed on core biopsy, as about half of the DCIS that were upgraded to invasive cancer in the subsequent resection specimen showed non-high grade nuclear atypia [10]. Therefore, the CRS should also be determined in core biopsies with pure DCIS, to investigate whether it can predict invasive carcinoma in subsequent resection specimens. This is of particular importance as active surveillance is currently investigated in several noninferiority trials [7-9].

We hypothesize that stromal changes are likely to reflect the ability of DCIS to evolve into frankly invasive breast cancer. Increased stromal inflammation might be attracted by an increased mutational load of some DCIS, which evokes immune responses by causing neo-antigen formation [39]. Stromal inflammatory cells may co-operate with cancer-associated fibroblasts in the periductal stroma to prepare the peri-tumoral microenvironment for subsequent invasion [40].

\section{Conclusions}

We advocate 2-tier histopathological assessment for recurrence risk stratification after BCS for DCIS, since we have shown its prognostic potential in this retrospective longitudinal cohort of 211 patients. We propose a combined risk score (CRS) to discern low risk from high risk patients, based on the presence of moderate to extensive stromal inflammation, myxoid stromal architecture and high nuclear grade. Future research should validate this CRS prospectively to investigate whether radiotherapy can be safely omitted in patients who are at low risk for developing recurrence. Accordingly, the CRS might aid to tailor future treatment of DCIS patients.

\section{Ethics approval and consent to participate}

This anonymized study was approved of by the ethics committee of the Leuven University Hospitals (locally registered as S56265/ML10212), and was granted dispensation from consent.

\section{Availability of data and materials}

All data generated and/or analyzed during the current study are available from the corresponding author on reasonable request.

\section{Conflicts of interest statement}

The authors declare no conflicts of interest.

\section{Funding}

The authors declare no sources of funding.

\section{CRediT authorship contribution statement}

Mieke Van Bockstal: Conceptualization, Data curation, Formal analysis, Project administration, Methodology, Writing the original draft, Writing - review and editing. Kathleen Lambein: Conceptualization, Formal analysis, Investigation, Methodology, Writing - review and editing. Ann Smeets: Data curation, Resources, Writing - review and editing. Laurence Slembrouck: Data curation, Writing - review and editing. Patrick Neven: Data curation, Writing - review and editing. Ines Nevelsteen: Data curation, Writing - review and editing. Caroline Weltens: Data curation, Writing - review and editing. Erik Van Limbergen: Data curation, Writing - review and editing. MarieRose Christiaens: Data curation, Writing - review and editing. Chantal Van Ongeval: Data curation, Writing - review and editing. Hans Wildiers: Data curation, Writing - review and editing. Louis Libbrecht: Conceptualization, Methodology, Writing - review and editing, Supervision. Giuseppe Floris: Conceptualization, Methodology, Writing - review and editing, Supervision.

\section{Acknowledgements}

Ms. Kathleen Van den Eynde is gratefully acknowledged for excellent technical assistance.

\section{Appendix}

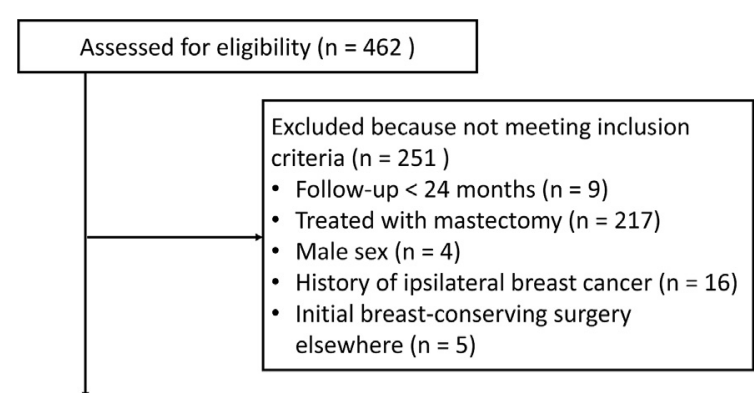

Eligible for retrospective study $(n=211)$

Figure A.1. Flow chart with exclusion criteria for this retrospective study. This flow chart indicates the number of eligible patients, the number of excluded patients because of not meeting the inclusion criteria, and the number of included patients in the final retrospective study. 

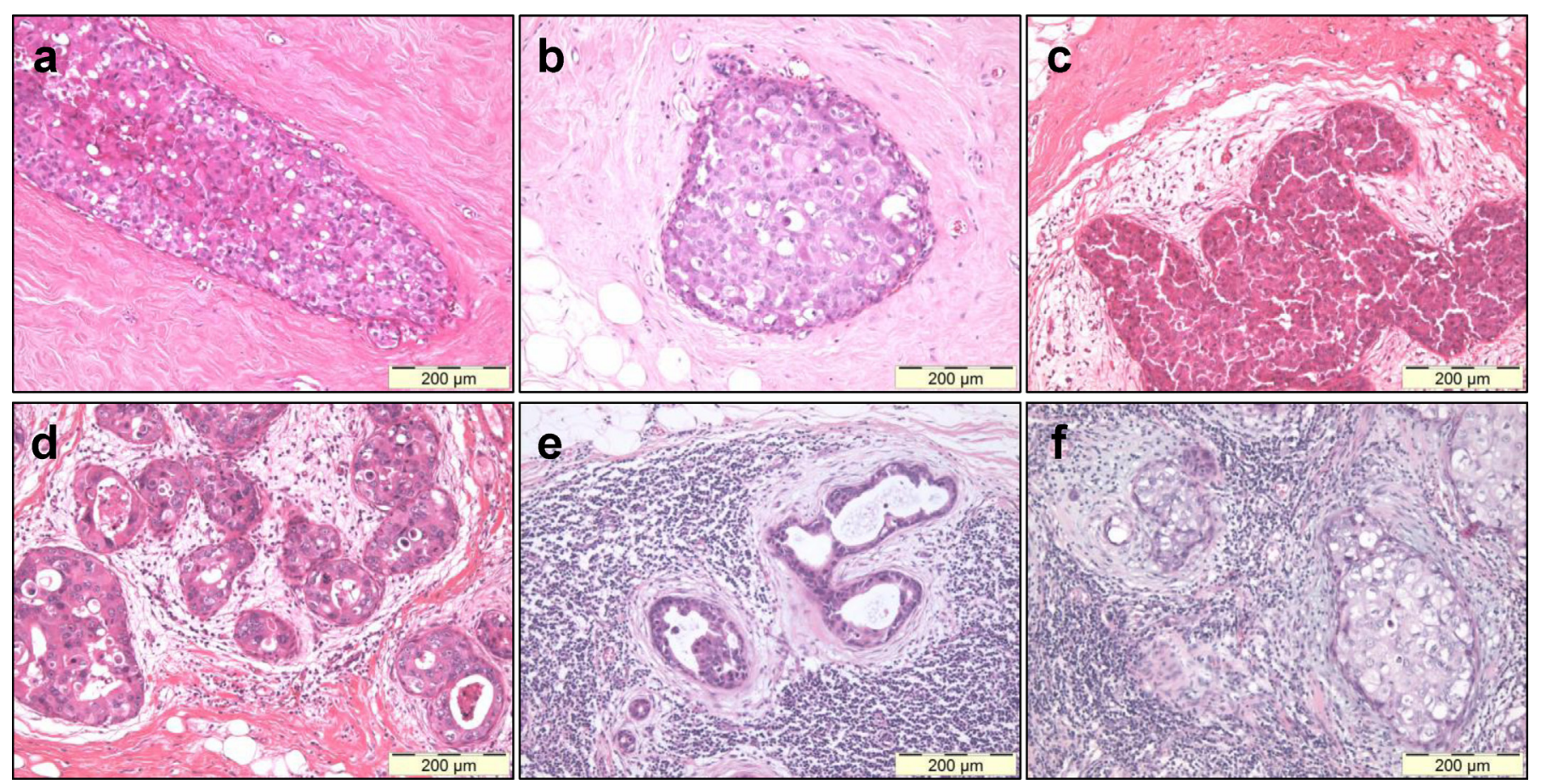

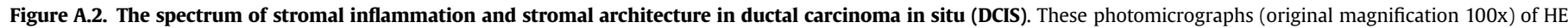

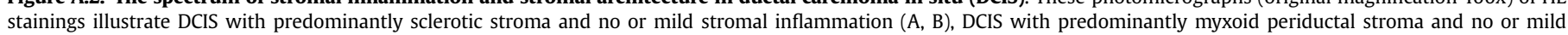
stromal inflammation (C, D), and DCIS with predominantly myxoid periductal stroma and moderate to extensive stromal inflammation (E, F). 2

\section{Appendix A. Supplementary data}

Supplementary data to this article can be found online at https://doi.org/10.1016/j.ejso.2018.11.005.

\section{References}

[1] Pang JM, Gorringe KL, Fox SB. Ductal carcinoma in situ - update on risk assessment and management. Histopathology 2016;68:96-109.

[2] Yeong J, Thike AA, Tan PH, Iqbal J. Identifying progression predictors of breast ductal carcinoma in situ. J Clin Pathol 2017;70:102-8.

[3] Agahozo MC, Hammerl D, Debets R, Kok M, van Deurzen CHM. Tumor-infiltrating lymphocytes and ductal carcinoma in situ of the breast: friends or foes? Mod Pathol 2018;31(7):1012-25.

[4] Maxwell AJ, Clements K, Hilton B, et al. Risk factors for the development of invasive cancer in unresected ductal carcinoma in situ. Eur J Surg Onco 2018;44:429-35.

[5] Sanders ME, Schuyler PA, Simpson JF, Page DL, Dupont WD. Continued observation of the natural history of low-grade ductal carcinoma in situ reaffirms proclivity for local recurrence even after more than 30 years of follow-up. Mod Pathol 2015;28:662-9.

[6] Collins LC, Tamimi RM, Baer HJ, Connolly JL, Colditz GA, Schnitt SJ. Outcome of patients with ductal carcinoma in situ untreated after diagnostic biopsy: results from the Nurses' Health Study. Cancer 2005;103:1778-84.

[7] https://dcisoptions.org/comet. Accessed on 26 September 2018.

[8] Elshof LE, Tryfonidis K, Slaets L, et al. Feasibility of a prospective, randomised, open-label, international multicentre, phase III, non-inferiority trial to asses the safety of active surveillance for low risk ductal carcinoma in situ - the LORD study. Eur J Canc 2015:51:1497-510.

[9] Francis A, Thomas J, Fallowfield L, et al. Addressing overtreatment of screen detected DCIS; the LORIS trial. Eur J Canc 2015;51:2296-303.

[10] Alexander M, Beyda J, Nayak A, Jaffer S. Not all ductal carcinomas in situ are created IDLE (Indolent Lesions of Epithelial Origin). Arch Pathol Lab Med 2018. https://doi.org/10.5858/arpa.2017-0366-OA. In press.

[11] Van Bockstal M, Lambein K, Gevaert O, et al. Stromal architecture and periductal decorin are potential prognostic markers for ipsilateral locoregiona recurrence in ductal carcinoma in situ of the breast. Histopathology 2013;63(4):520-33.

[12] Van Bockstal M, Baldewijns M, Colpaert C, et al. Dichotomous histopathological assessment of ductal carcinoma in situ of the breast results in substantial inter-observer concordance. Histopathology 2018. https://doi.org/ 10.1111/his.13741. In press.

[13] Silverstein MJ. The University of Southern California/Van Nuys prognostic index for ductal carcinoma in situ of the breast. Am J Surg 2003;186:337-43.

[14] Han K, Nofech-Mozes S, Narod S, et al. Expression of HER2neu in ductal carcinoma in situ is associated with local recurrence. Clin Oncol 2012;24:183-9.

[15] Wapnir IL, Dignam JJ, Fisher B, et al. Long-term outcomes of invasive ipsilateral breast tumor recurrences after lumpectomy in NSABP B-17 and B-24 randomized clinical trials for DCIS. J Natl Cancer Inst 2011;103:478-88.

[16] McShane LM, Altman DG, Sauerbrei W, et al. REporting recommendations for tumour MARKer prognostic studies (REMARK). Eur J Canc 2005;41:1690-6.

[17] Lester SC, Bose S, Chen YY, et al. Protocol for the examination of specimens from patients with ductal carcinoma in situ of the breast. Arch Pathol Lab Med 2009;133:15-25.

[18] Pinder SE, Duggan C, Ellis IO, et al. A new pathological system for grading DCIS with improved prediction of local recurrence: results from the UKCCCR/ANZ DCIS trial. Br J Canc 2010;103:94-100.

[19] Van Bockstal M, Lambein K, Denys H, et al. Histopathological characterization of ductal carcinoma in situ (DCIS) of the breast according to HER2 amplification status and molecular subtype. Virchows Arch 2014;465(3):275-89.

[20] Van Bockstal M, Libbrecht L, Floris G, Lambein K. The Baader-Meinhof phenomenon in ductal carcinoma in situ of the breast. Histopathology 2016;69: $522-3$.

[21] Cambra MJ, Farrús B, Moreno F, et al. Management of breast ductal carcinoma in situ in Catalonia, Spain: results from the Grup Oncologic Calala-OccitaCatalonia survey with 9-year follow up. Breast 2017:35:196-202.

[22] Kajiwara Y, Ueno H, Hashiguchi Y, Mochizuki H, Hase K. Risk factors of nodal involvement in T2 colorectal cancer. Dis Colon Rectum 2010;53:1393-9.

[23] Ueno H, Shinto E, Hashiguchi Y, et al. In rectal cancer, the type of desmoplastic response after preoperative chemoradiotherapy is associated with prognosis. Virchows Arch 2015;466:655-63.

[24] Wernicke M, Piñeiro LC, Caramutti D, et al. Breast cancer stromal myxoid changes are associated with tumor invasion and metastasis: a central role for hyaluronan. Mod Pathol 2003;16:99-107.

[25] Siziopikou KP. Ductal carcinoma in situ of the breast: current concepts and future directions. Arch Pathol Lab Med 2013;137:462-6.

[26] Shatat L, Gloyeske N, Madan R, O'Neil M, Tawfik O, Fan F. Microinvasive breast carcinoma carries an excellent prognosis regardless of the tumor characteristics. Hum Pathol 2013;44:2684-9.

[27] Gorringe KL, Fox SB. Ductal carcinoma in situ biology, biomarkers, and diagnosis. Front Oncol 2017;7:248.

[28] Pruneri G, Lazzeroni M, Bagnardi V, et al. The prevalence and clinical relevance of tumor-infiltrating lymphocytes (TILs) in ductal carcinoma in situ of the breast. Ann Oncol 2017;28:321-8.

[29] Hendry S, Salgado R, Gevaert T, et al. Assessing tumor-infiltrating lymphocytes in solid tumors: a practical review for pathologists and proposal for a standardized method from the international immunooncology biomarkers working group: Part 1: assessing the host immune response, TILs in invasive 
breast carcinoma and ductal carcinoma in situ, metastatic tumor deposits and areas for further research. Adv Anat Pathol 2017;24:235-51.

[30] Toss MS, Miligy I, Al-Kawaz, et al. Prognostic significance of tumor-infiltrating lymphocytes in ductal carcinoma in situ of the breast. Mod Pathol 2018;31(8): $1226-36$.

[31] Lopez-Garcia MA, Geyer FC, Lacroix-Triki M, Marchió C, Reis-Filho JS. Breast cancer precursors revisited: molecular features and progression pathways. Histopathology 2010;57:171-92.

[32] Hannemann J, Velds A, Halfwerk JB, Kreike B, Peterse JL, van de Vijver MJ Classification of ductal carcinoma in situ by gene expression profiling. Breast Cancer Res 2006;8:R61.

[33] Balleine RL, Webster LR, Davis S, et al. Molecular grading of ductal carcinoma in situ of the breast. Clin Canc Res 2008:14:8244-52.

[34] Hoorntje LE, Schipper ME, Peeters PH, Bellot F, Storm RK, Borel Rinkes IH. The finding of invasive cancer after a preoperative diagnosis of ductal carcinomain-situ: causes of ductal carcinoma-in-situ underestimates with stereotactic 14-gauge needle biopsy. Ann Surg Oncol 2003;10:748-53.
[35] Doebar SC, de Monyé C, Stoop H, Rothbarth J, Willemsen SP, van Deurzen CH. Ductal carcinoma in situ diagnosed by breast needle biopsy: predictors of invasion in the excision specimen. Breast 2016;27:15-21.

[36] Walters LL, Pang JC, Zhao L, Jorns JM. Ductal carcinoma in situ with distorting sclerosis on core biopsy may be predictive of upstaging on excision. Histopathology 2015;66:577-86.

[37] Al Nemer AM. Histologic factors predicting invasion in patients with ducta carcinoma in situ (DCIS) in the preoperative core biopsy. Pathol Res Pract 2017;213:429-34

[38] Yang M, Moriya T, Oguma M, et al. Microinvasive ductal carcinoma (T1mic) of the breast. The clinicopathological profile and immunohistochemical features of 28 cases. Pathol Int 2003;53:422-8.

[39] Van Bockstal M, Libbrecht L, Floris G, Lambein K, Pinder S. Stromal inflammation, necrosis and HER2 overexpression in ductal carcinoma in situ of the breast: another causality dilemma? Ann Oncol 2017;28:2317.

40] Van Bockstal M, Libbrecht L, De Wever O. Fibroblast-induced matrix remodeling paves the path for invasion. Cell Cycle 2015;14:793-4. 